\title{
A Receptor Component of the Chloroplast Protein Translocation Machinery
}

\author{
Stephan Hirsch, ${ }^{\star}$ Eva Muckel, ${ }^{\star}$ Frank Heemeyer, \\ Gunnar von Heijne, Jürgen Soll $\dagger$
}

The chloroplast outer envelope protein OEP86 functions as a receptor in precursor protein translocation into chloroplasts. Sequence analysis suggests that the precursor of OEP86 is directed to the chloroplast outer envelope by a cleavable, negatively charged, and unusually long amino-terminal peptide. This presequence is unlike other potential targeting signals and suggests the existence of another membrane insertion pathway. Insertion of precursor OEP86 required the hydrolysis of adenosine triphosphate and the existence of surface exposed chloroplast membrane components, and it was not competed by another precursor protein destined for the internal plastid compartments.

The protein import machinery of the outer envelope of pea chloroplasts can be isolated as one functional active unit $(1,2)$. The ability to recognize and translocate precursor proteins is retained in the isolated import complex $(2,3)$. The main constituents of the import complex are the proteins OEP86, OEP75, OEP34, and a heat shock cognate 70 homolog $(1-3)$. OEP86 is involved very early in the pathway and is a protease-sensitive component of the receptor unit $(4,5)$. The biological functions of single components of the chloroplast import machinery have not been characterized (6-9).

Antibodies raised in rabbits to OEP86, either polyclonal immunoglobulin $\mathrm{G}$ or Fab fragments, were able to inhibit import and to decrease but not eliminate binding of the precursor (pre-) of the small subunit of ribulose-1,5-bisphosphate carboxylase oxygenase (SSU), a stroma-localized protein (10). Fab fragments of antibodies to OEP75 did not inhibit pre-SSU binding or import (Fig. 1). OEP75 is located in the outer envelope, where it is protease-resistant $(11,12)$ and serves as a component of the translocation apparatus $(4,9)$. These data as well as crosslinking studies $(4,5)$ indicate that OEP86 is required for import of pre-SSU. OEP86 might also serve in the same function for other plastidial precursor proteins, which share a similar translocation mechanism $(9,13)$.

A full-length complementary DNA (cDNA) clone (pisa 86a) was isolated from a cDNA library synthesized from polyadenylated mRNA of etiolated pea leaves [pisa 86a; European Molecular Biology Laboratory (EMBL) accession number Z31588] (Fig.

S. Hirsch, E. Muckel, F. Heemeyer, J. Soll, Botanisches Institut, Universität Kiel, Olshausenstraße 40, 24098 Kiel, Germany.

G. von Heijne, Karolinska Institute Center for Structural Biochemistry, NOVUM, S-14157 Huddinge, Sweden.

*The first two authors contributed equally to this work. †To whom correspondence should be addressed.
2). $\mathrm{NH}_{2}$-terminal and internal peptide sequence information confirms that pisa $86 a$ codes for OEP86 from pea (Fig. 2). The long open reading frame in front of the $\mathrm{NH}_{2}-$ terminal protein sequence may represent a targeting signal for OEP86. Translation could begin at either of two methionines at

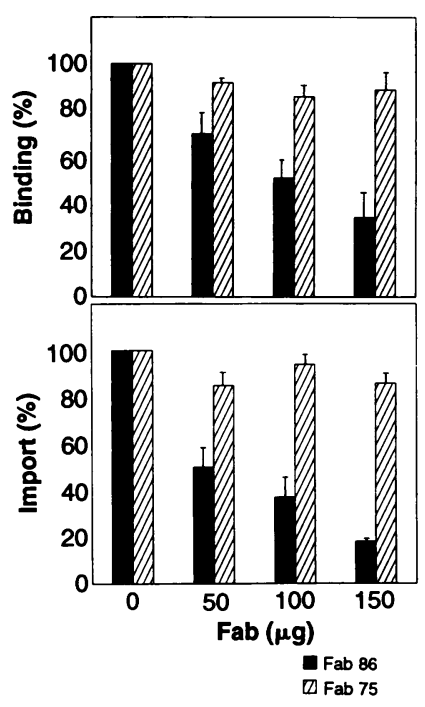

Fig. 1. Binding and import of pre-SSU is inhibited specifically by antibodies to OEP86 Fab fragments. The polyclonal antisera were raised in rabbits against SDS-PAGE-purified polypeptides and have been described before $(3,21)$. Intact chloroplasts were incubated in $300 \mu$ l of import buffer (1) for $30 \mathrm{~min}$ at $4^{\circ} \mathrm{C}$ with different amounts of $\mathrm{Fab}$ fragments derived from purified immunoglobulin G of OEP75 and OEP86 antisera (31). Organelles were purified from the preincubation mixture, and chloroplasts equivalent to $10 \mu \mathrm{g}$ of chlorophyll were used in a standard binding (50 $\mu \mathrm{M}$ ATP) or import ( $3 \mathrm{mM}$ ATP) reaction with the use of ${ }^{35}$ S-labeled pre-SSU translation product. Binding inhibition was quantified by laser densitometry of exposed $x$-ray films (mean of three experiments). Import inhibition, the appearance of mature SSU inside the organelle, was quantified as above (mean of three experiments; standard error is indicated). amino acid positions 1 and 31 . The calculated molecular weight from translation initiation at amino acid 1 is $96 \mathrm{kD}$, and from amino acid 31 is $93 \mathrm{kD}$. The in vitro translation product of pisa $86 \mathrm{a}$ has an apparent size of $98 \mathrm{kD}$, which suggests that the first methionine is the start for precursor OEP86 (pre-OEP86). The protein sequences of OEP86 and OEP34 share 34\% similarity, and an additional $25 \%$ of amino acids are conservative replacements (Fig. 2). OEP34 is another component of the chloroplast outer envelope import complex $(3,14)$.

An adenosine triphosphate (ATP) binding site or P loop consensus sequence (15) is present in OEP86 at amino acid positions 245 to 252, which suggests that ATP influences the receptor-precursor interaction. The cell adhesion motif RGD (16) was detected at positions 805 to 807 . Hydrophobicity analysis did not reveal stretches of $\infty$ amino acids sufficiently long to span the lipid bilayer. The putative presequence is 146 amino acids long and carries considerable negative charges, in contrast to transit peptides that direct proteins to the stroma 운 of higher plant chloroplasts. Stromal transit peptides that are much shorter lack acidic residues and are rich in hydroxylated amino acids $(13,17,18)$. The processing site of pre-OEP86 (Fig. 2) is unlike that used by the soluble stromal peptidase $(13,18)$.

OEP86 was very susceptible to proteolysis either in vitro or in situ. It is converted to a $52-\mathrm{kD}$ fragment by exogenous added 0 protease and is completely accessible to protease when the membranes are solubilized by detergent (Fig. 3A) (19). OEP86 is also $\varepsilon$ partially degraded by endogenous proteolyt- 은 ic activity to yield a $52-\mathrm{kD}$ fragment during organelle and membrane isolation (20). The amino acid sequence of the $52-\mathrm{kD}$ 。 fragment generated by the protease thermolysin (compare Fig. 3A, lane 2) starts at residue 474 (Fig. 2), which demonstrates that the $\mathrm{NH}_{2}$-terminus of OEP86 is exposed on the chloroplast surface and could carry the functional domains for precursor protein recognition (21).

Pre-OEP86 synthesized from pisa 86 a by in vitro transcription and translation in the presence of radiolabeled methionine has an apparent molecular size on SDS-polyacrylamide gel electrophoresis (SDS-PAGE) of $98 \mathrm{kD}$. It binds to intact purified chloroplasts under standard import conditions ( 1 , 2 ) and is processed to an $86-\mathrm{kD}$ form (Fig. 3B). The processed form of imported OEP86 exhibited the same electrophoretic mobility as the endogenous OEP86, as determined by protein immunoblot of an import assay after transfer to nitrocellulose filters and autoradiography (22). Only imported OEP 86 could be converted by exogenous protease to the $52-\mathrm{kD}$ fragment (Fig. $3 \mathrm{~B})$, whereas the pre-OEP86 translation 
product is completely susceptible to protease (23). Translocated $96-\mathrm{kD}$ pre-OEP86, the processed $86-\mathrm{kD}$ OEP86, and the $52-\mathrm{kD}$ fragment were recovered in the insoluble membrane pellet fraction after treatment of the chloroplasts with large amounts of salt or high $\mathrm{pH}$ (Fig. 3B). In contrast, the preOEP86 translation product was detected in the soluble fraction after extraction with high pH (Fig. 3B). We conclude therefore that in vitro-translocated OEP86 is correctly integrated and folded in its target membrane. Integration of pre-OEP86 into the membrane seemed to precede processing, as most of pre-OEP86 was also detected in a location resistant to $\mathrm{Na}_{2} \mathrm{CO}_{3}$ extraction (Fig. 3B). Chloroplast surface-bound preSSU is largely recovered in the soluble protein fraction after $\mathrm{Na}_{2} \mathrm{CO}_{3}$ treatment (Fig. $3 \mathrm{C}$ ), which indicates that binding of a pre-

Fig. 2. Sequence analysis of preOEP86 from pea as derived from the CDNA clone pisa $86 a$ (EMBL accession number Z31588). Abbreviations for the amino acid residues are A, Ala; C, Cys; D, Asp; E, Glu; F, Phe; G, Gly; H, His; I, lle; K, Lys; L, Leu; M, Met; N, Asn; P, Pro; Q, Gln; R, Arg; S, Ser; T, Thr; V, Val; W, Trp; and Y, Tyr. Both strands were sequenced. $\mathrm{NH}_{2}$-terminal and internal peptide sequences of endoprotease Glu-C fragments of OEP86 were obtained by Edman degradation and are underlined. The processing site of preOEP86 is indicated by an arrowhead $(\boldsymbol{\nabla})$; the beginning of the 52 $\mathrm{kD}$ fragment is also indicated $(\nabla$, position 474). The 52-kD fragment was purified by SDS-PAGE from protease (thermolysin)treated pea chloroplast outer envelopes (20). The ATP-binding site is indicated by asterisks (positions 245 to 252). OEP86 has sequence homology to OEP34

from pea; a partial OEP34 amino acid sequence is shown underneath the pre-OEP86 sequence (asterisks indicate identities, dots conservative exchanges). Dashes were introduced into the OEP34 sequence for the best alignment. cursor protein to the chloroplast import machinery per se does not render it resistant to alkaline.

Protein translocation into chloroplasts occurs simultaneously through the import machineries of both the inner and outer envelopes $(5,24)$. Precursor proteins-for example, pre-SSU-span both envelope membranes while in transit through the membranes $(5,24)$. The existence of stationary translocation contact sites has been proposed for chloroplasts $(4,6,24)$. We therefore wanted to determine the exact localization of imported OEP86 in the different chloroplast membranes in comparison to OEP75 and IEP110 as marker proteins for the outer and inner envelopes, respectively (25). Outer and inner envelope membranes were isolated from intact chloroplasts after import assays and separated on linear sucrose density gradients. Although OEP75, an integral membrane protein and component of the translocation machinery $(4,9)$, was detected by immunoblot analysis in the low-density fractions of the gradient, where purified outer envelope membranes would be expected $(25,26)$, IEP110 was found in higher density fractions in the gradient, well separated from gradient fractions containing OEP75 (Fig. 3D). Both imported OEP86 and OEP75 were found only in the low-density fractions of the gradient (Fig. 3D), which indicates that they are located in the outer envelope, as is the unlabeled protein in situ $(25,26)$. Translocation contact sites would be expected in gradient fractions of intermediate density (26). The gradient density analysis demonstrated that two polypeptides involved in protein transport, OEP86 and
Fig. 3. Characteristics of OEP 86 and its precursor in chloroplasts. (A) Effect of protease on OEP86 in situ. Purified outer envelope membranes (10 $\mu \mathrm{g}$ of protein) from pea chloroplasts were either not treated (lane 1) or treated (lane 2) with thermolysin (Th, $1 \mu \mathrm{g}$ ) (20) in the presence of detergent ( $1 \%$ Triton $X-100$, lane 3). A Coomassie brilliant blue SDS-PAGE is shown. The positions of OEP86 and the $52-\mathrm{kD}$ fragment $\left(52^{*}\right)$ are indicated. (B) The preOEP86 translation product (TL) is imported into intact chloroplasts (1) and processed to its mature form OEP86 (lane 1 , contains $1 / 10$ of pre-OEP86 added to the translocation reactions), shown here in a fluorograph of an SDSPAGE. Organelles were either treated without or with the protease thermoly$\sin (\mathrm{Th})$ after import (1), respectively, and subsequently extracted with $1 \mathrm{M}$ $\mathrm{NaCl}$ or $0.1 \mathrm{M} \mathrm{Na}_{2} \mathrm{CO}_{3}$. The extraction mixture was separated into supernatant $(\mathrm{S})$ and membrane pellet $(\mathrm{P})$. The translation product was subjected to an identical $\mathrm{Na}_{2} \mathrm{CO}_{3}$ treatment. (C) Chloroplast-bound pre-SSU is extractable by alkaline treatment, shown here in a fluorograph. Chloroplasts were incubated with ${ }^{35}$ S-labeled pre-SSU under binding conditions (1) and repurified and subjected to extraction in the absence or presence of $\mathrm{Na}_{2} \mathrm{CO}_{3}$ as in (B). (D)

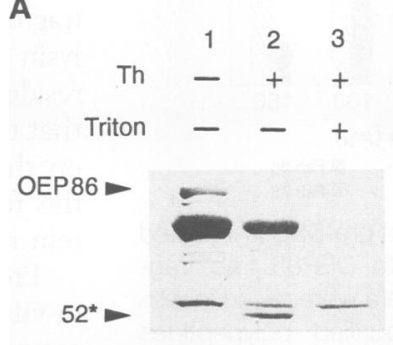

C

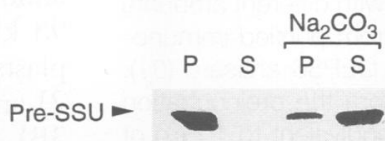

B

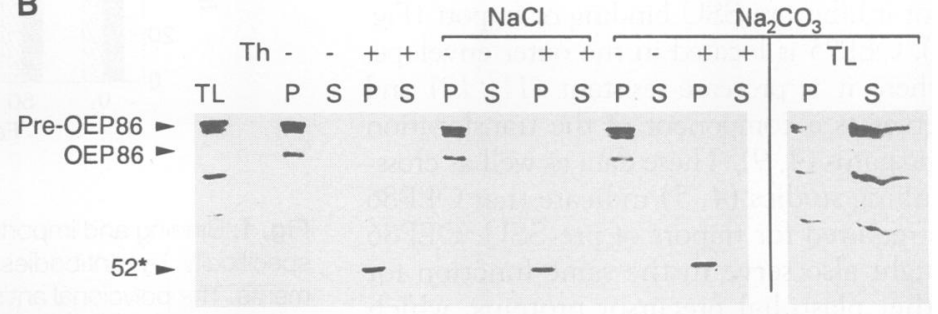

D

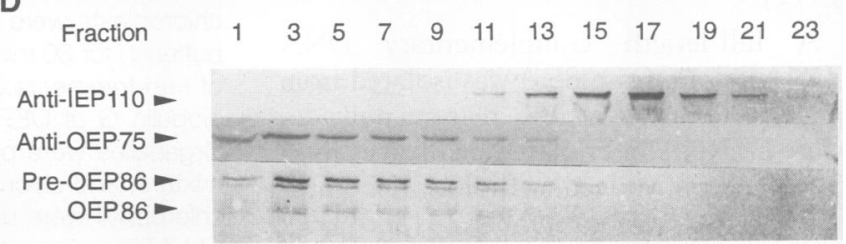

Precursor and mature OEP86 are localized in the outer envelope. After import of pre-OEP86 as in (B), five reactions were combined and envelope membranes were separated and purified after hypertonic lysis of the organelles on linear sucrose density gradients $(25,26)$. Fractions were analyzed by immunoblotting with the use of either IEP110 or OEP75 antiserum (anti-IEP110 and anti-OEP75). In parallel, ${ }^{35}$ S-labeled pre-OEP86 and mature OEP86 were detected by SDS-PAGE and fluorography. 
OEP75, are mostly if not exclusively found in the outer chloroplast envelope. We suggest that the protein translocation unit of the outer chloroplast envelope is independent of, but may cooperate with that of, the inner membrane $(2,9)$. For unknown reasons, the ratio between processed mature OEP86 and pre-OEP86 varied (Fig. 3, C and D). In general, between 5 and $10 \%$ of the added pre-OEP86 translation product was recovered together with the chloroplasts after a translocation experiment.

Other OEPs as yet identified from plastids and mitochondria do not possess a cleavable $\mathrm{NH}_{2}$-terminal precursor sequence (presequence) $(17,27,28)$. The translocation requirements of OEP86 might therefore be different from those of other OEPs, such as OEP7 from spinach (27) and OEP14 from pea (28). Translocation of pre-OEP86 requires both hydrolysis of ATP for productive insertion and the presence of protease-sensitive chloroplast surface components for binding (Fig. 4A). Excess (0.1 $\mu \mathrm{M})$ unlabeled pre-SSU severely blocks import of the pre-SSU translation product into chloroplasts (Fig. 4B), but it barely affects the integration and processing of pre-OEP86 in the outer chloroplast envelope.

These results indicate that there are different translocation pathways for proteins destined for plastids, which contain cleavable presequences. To elucidate the possible role of the OEP86 presequence, we either partially or completely deleted the presequence. An intermediate-size OEP86 (iOEP86), which contains about half of the presequence, was able to bind to intact chloroplasts; however, the subsequent processing to OEP86 was impaired. This could indicate that either the insertion pathway or the processing is impaired as a result of

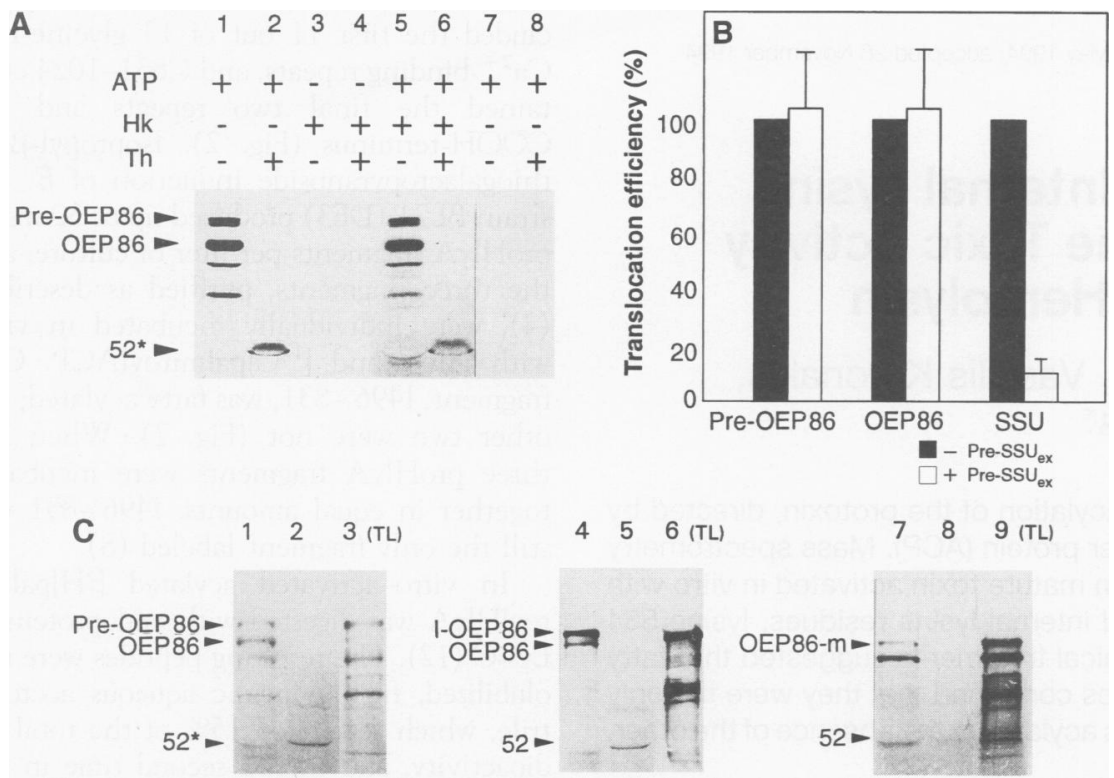

Fig. 4. Translocation behavior of pre-OEP86 in intact chloroplasts. (A) Import of pre-OEP86 requires ATP and protease-sensitive chloroplast surface components. Import was tested in the presence of $3 \mathrm{mM} \mathrm{ATP}$ (lanes 1 and 2) or after depletion of ATP by a glucose-hexokinase $(\mathrm{HK})$ trap $(0.5 \mathrm{mM}$ glucose and $10 \mathrm{U}$ of hexokinase) (32) (lanes 3 and 4). ATP was re-added to a concentration of $3 \mathrm{mM}$ at 10 and $20 \mathrm{~min}$ during the import reaction (lanes 5 and 6). Organelles were either not treated or treated with thermolysin after import as indicated. In lanes 7 and 8 , chloroplasts were pretreated with $750 \mu \mathrm{g}$ of thermolysin per milligram of chlorophyll for $30 \mathrm{~min}$ on ice before the import reaction. Protease-treated organelles were purified by density gradient centrifugation and washed twice in medium containing 10 mM EDTA. The final pellet was resuspended in import buffer (1) and used for translocation assays. Further manipulations are as indicated. The data presented in lanes 7 and 8 were obtained from a separate experiment. (B) Pre-OEP86 uses a different translocation pathway than pre-SSU. Pre-SSU (33) was overexpressed in Escherichia coli cells and recovered as insoluble protein from inclusion bodies (34). The overexpressed

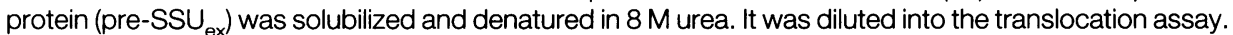
The final urea concentration was $80 \mathrm{mM}$, which was also present in controls without pre-SSU $\mathrm{ex}_{\text {. In }}$ addition, radiolabeled pre-SSU and pre-OEP86 were added, respectively. The reaction was started by the addition of chloroplasts. Experiments were done under conditions optimal for pre-SSU import into chloroplasts (that is, $3 \mathrm{mM} \mathrm{ATP}$ ), which do not allow measurement of pre-SSU binding $(1,29)$. Products were analyzed by SDS-PAGE and fluorography. Radiolabeled proteins-that is, pre-OEP86, OEP86, and SSU-were quantified by a laser densitometer. A mean of five experiments is shown, and standard error is indicated. (C) Effect of deletions of the presequence of pre-OEP86. Pre-OEP86, i-OEP86 (35), and OEP86-m (35) translation products were added to intact chloroplasts, respectively, under standard import conditions. Lanes 3,6 , and 9 show $1 / 10$ of the amount of translation product (TL) added to the translocation assay. Chioroplasts were either not treated (lanes 1, 4, and 7) or treated (lanes 2, 5, and 8) with thermolysin (1) after completion of the experiment. the partial deletion of the transit peptide. The small amount of the processed form of imported i-OEP86 is converted by protease to the $52-\mathrm{kD}$ fragment. In vitro-synthesized OEP86 (OEP86-m), which starts at amino acid position 150 , three amino acids behind the processing site, could still adhere to chloroplasts. However, this interaction did not result in the insertion of OEP86-m into the membrane, because we could not detect the $52-\mathrm{kD}$ fragment after protease treatment. The presequence of OEP86 seems to fulfill an essential role by keeping the in vitro-synthesized precursor protein on an efficient and specific translocation pathway.

Translocation of precursor proteins into chloroplasts requires ATP for precursor binding (29). OEP86 itself probably requires ATP for function, as it contains a conserved ATP binding site and is phosphorylated in situ with a Michaelis constantoo for ATP in the micromolar range (30), similar to that required for the binding of precursor proteins. The processing peptidase for pre-OEP86 has yet to be identified, but it should be associated with the plastid운 outer envelope fraction, because the $\mathrm{NH}_{2}-\frac{c}{0}$ terminus of OEP86 seems to be exposed to 0 the cytosol and it is unlikely that it crosses both envelope membranes to reach the stroma for processing.

\section{REFERENCES AND NOTES}

1. K. Waegemann and J. Soll, Plant J. 1, 149 (1991). 2. J. Soll and K. Waegemann, ibid. 2, 253 (1992).

3. K. Waegemann and J. Soll, in Molecular Mechanisms of Membrane Traffic, D. J. Morre, J. J. M. Bergeron, K. E. Howell, Eds. (NATO ASI Series, Springer-Verlag Berlin, Heidelberg, 1993), vol. 74, pp. 101-104.

4. S. E. Perry and K. Keegstra, Plant Cell 6, 93 (1994).

5. H. Alefsen, K. Waegemann, J. Soll, J. Plant Physiol. \} 144, 339 (1994).

6. D. Pain, Y. S. Kanwar, G. Blobel, Nature 331, 232 O (1988).

7. D. J. Schnell, G. Blobel, J. Pain, J. Cell Biol. 111, 1825 (1990).

8. U.-I. Flügge et al., Nature 353, 364 (1991).

9. J. Soll and H. Alefsen, Physiol. Plant. 87, 433 (1993).

10. The reduction of pre-SSU binding is probably less pronounced than inhibition of import, because preSSU binds to a certain extent nonspecifically to the chloroplast surface $(8,13)$.

11. J. Joyard et al., J. Biol. Chem. 258, 10000 (1983).

12. K. Cline, M. Werner-Washburne, J. Andrews, K Keegstra, Plant Physiol. 75, 675 (1984).

13. A. D. de Boer and P. J. Weisbeek, Biochim. Biophys. Acta 1071, 221 (1991).

14. M. Seedorf, K. Waegemann, J. Soll, Plant J., in press. The OEP34 sequence has been deposited under EMBL accession number Z28341.

15. Y. Kaziro, H. Itoh, T. Kozasa, M. Nakafuku, T. Satok, Annu. Rev. Biochem. 60, 349 (1991)

16. S. E. d'Souza, M. H. Ginsberg, E. F. Plow, Trends Biochem. Sci. 16, 246 (1991).

17. F.-U. Hartl, M. Pfanner, D. W. Nicholson, W. Neupert, Biochim. Biophys. Acta 988, 1 (1989).

18. G. von Heijne, J. Stepphuhn, R. G. Herrmann, Eur. J. Biochem. 180, 535 (1989).

19. Protease treatment of intact chloroplasts yields always slightly less of the 52-kD fragment than expected for the amount of OEP86 present at the beginning of the treatment. This indicates that proteolysis could proceed further under the in vitro conditions applied. Furthermore, preliminary evidence indicates that 
carbonate-resistant pre-OEP86 does not yield the $52-\mathrm{kD}$ fragment-that is, folding and integration into the envelope seem incomplete at this stage.

20. K. Waegemann, S. Eichacker, J. Soll, Planta 187, 89 (1992).

21. Protease treatment of intact chloroplasts yields only one membrane-associated OEP86 breakdown product, namely the $52-\mathrm{kD}$ fragment. The $\mathrm{NH}_{2}$-terminal portion of OEP86 seems unprotected from proteolysis by association with the outer envelope membrane.

22. A standard pre-OEP86 translocation reaction was separated by SDS-PAGE and electrophoretically transferred to nitrocellulose filters (20). The filters analyzed by protein immunoblot were with an OEP86 antiserum and stained with the use of the alkaline phosphatase color reaction (20). The nitrocellulose filter was subsequently subjected to autoradiography. The band labeled by the OEP 86 antiserum coincided completely with the labeled band on the $x$ ray film.

23. Pre-OEP86 translation product was treated with $1 \mu \mathrm{g}$ of thermolysin for $10 \mathrm{~min}$ on ice (20). The radioactive labeled pre-OEP 86 and polypeptides of lower molecular mass were completely degraded.

24. D. J. Schnell and G. J. Blobel, J. Cell Biol. 120, 103 (1993).

25. M. A. Block, A.-J. Dorne, J. Joyard, R. Douce, J. Biol. Chem. 258, 13281 (1983)

26. K. Keegstra and A. E. Youssif, Methods Enzymol. 118, 316 (1986)
27. M. Salomon, K. Fischer, U.-I. Flügge, J. Soll, Proc. Natl. Acad. Sci. U.S.A. 87, 5778 (1990)

28. H.-M. Li, T. Moore, K. Keegstra, Plant Cell 3, 709 (1991).

29. L. J. Olsen, S. M. Theg, B. R. Selman, K. Keegstra, J. Biol. Chem. 264, 6724 (1989)

30. J. Soll, V. Berger, J. Bennett, Planta 177, 393 (1989).

31. E. Harlow and D. Lane, Antibodies: A Laboratory Manual (Cold Spring Harbor Laboratory, Cold Spring Harbor, NY, 1989).

32. C. Schindler, R. Hracky, J. Soll, Z. Naturforsch. 42c, 103 (1987).

33. R. R. Klein and M. E. Salvucci, Plant Physiol. 98, 546 (1992).

34. K. Waegemann, H. Paulsen, J. Soll, FEBS Lett. 261, 89 (1990).

35. Both i-OEP86 and OEP86-m were constructed from the original clone after restriction with Sac I and Pst I, respectively, and subcloned into the vector pGEM5Zf(+) (Promega). The open reading frame for i-OEP86 contained six additional amino acids (Met, His, Pro, Thr, Arg, and Trp) before the start of the original protein at amino acid 83. OEP86-m started three amino acid positions behind the proteolytic processing site (that is, at amino acid 150).

36. Supported in part by grants from the Deutsche Forschungsgemeinschaft (J.S.) and by a grant from the Swedish Natural Sciences Research Council (G.v.H.).

19 May 1994; accepted 28 November 1994

\title{
Fatty Acylation of Two Internal Lysine Residues Required for the Toxic Activity of Escherichia coli Hemolysin
}

\author{
Peter Stanley, Len C. Packman, Vassilis Koronakis, \\ Colin Hughes*
}

Hemolysin of Escherichia coli is activated by fatty acylation of the protoxin, directed by the putative acyl transferase HlyC and by acyl carrier protein (ACP). Mass spectrometry and Edman degradation of proteolytic products from mature toxin activated in vitro with tritium-labeled acyIACP revealed two fatty-acylated internal lysine residues, lysine 564 and lysine 690 . Resistance of the acylation to chemical treatments suggested that fatty acid was amide linked. Substitution of the two lysines confirmed that they were the only sites of acylation and showed that although each was acylated in the absence of the other, both sites were required for in vivo toxin activity.

Hemolysin (HlyA) secreted by pathogenic E. coli binds to mammalian cell membranes, disrupting cellular activities and causing cell lysis by pore formation $(1,2)$. The toxin is made as an inactive protoxin (proHlyA) that is activated intracellularly by the cosynthesized protein HlyC (3). The transformation of proHlyA to mature HlyA toxin is determined by fatty acylation directed by homodimeric HlyC, which uses only acylated acyl carrier protein as a fatty acid donor $(4,5)$. The mechanism, which is

\footnotetext{
P. Stanley, V. Koronakis, C. Hughes, Department of Pathology, Cambridge University, Tennis Court Road, Cambridge CB2 1QP, UK.

L. C. Packman, Department of Biochemistry, Cambridge University, Tennis Court Road, Cambridge CB2 1QW, UK.

*To whom correspondence should be addressed.
}

required for the activity of a family of membrane-targeted toxins, including leukotoxins of Pasteurella and Actinobacillus and the adenylate cyclase-hemolysin of Bordetella pertussis (6), does not conform to protein maturation processes such as $\mathrm{NH}_{2}$-terminal myristoylation of glycines and generation of $\mathrm{N}$-acyl diglyceride cysteines, acylation of internal residues through ester linkages, or $\mathrm{COOH}$-terminal glypiation (5). We have now defined the specific sites of the toxin fatty acylation in vitro and correlate them to the in vivo toxin activity.

During in vitro reactions containing only purified proHlyA, HlyC, and $\left[{ }^{14} \mathrm{C}\right]$ palmitoylACP (7), inactive proHlyA was converted efficiently to mature HlyA toxin (Fig. 1), with hemolytic activity and transfer of labeled fatty acid from ACP increasing in parallel and in direct proportion to the HlyC concentration. Hydroxylaminolysis and alkaline methanolysis of the reaction mixture released the labeled fatty acid from $\left[{ }^{14} \mathrm{C}\right]$ palmitoylACP but not from $\left[{ }^{14} \mathrm{C}\right]$ palmitoylHlyA (Fig. 1), and no labeled compounds of small molecular size were detected in the chloroform-methanol phase when active acylated toxin was treated alone (8). When $\left[{ }^{14} \mathrm{C}\right]$ palmitoylHlyA was treated with trifluoromethanesulfonic acid (TFMS), HlyA again remained labeled and again no labeled compounds of small molecular size were found in the extracting organic phase (Fig. 1). The data indicate that the fatty acid was linked covalently, not through an acyl ester bond or sugar linkage but most likely directly by an amide bond.

Three fragments spanning the 1024-residue proHlyA were generated in vivo from T7 expression vectors $(9-11)$. Fragment N1-520 included the entire hydrophobic membrane-spanning domain, I496-831 included the first 11 out of 13 glycine-rich $\mathrm{Ca}^{2+}$-binding repeats, and C831-1024 contained the final two repeats and the $\mathrm{COOH}$-terminus (Fig. 2). Isopropyl- $\beta$-Dthiogalactopyranoside induction of $E$. coli strain BL21 (DE3) produced up to $30 \mathrm{mg}$ of proHlyA fragments per liter of culture, and the three fragments, purified as described (4), were individually incubated in vitro with $\mathrm{HlyC}$ and $\left[{ }^{14} \mathrm{C}\right]$ palmitoylACP. One fragment, I496-831, was fatty acylated; the other two were not (Fig. 2). When the three proHlyA fragments were incubated together in equal amounts, I496-831 was still the only fragment labeled (8).

In vitro-activated acylated $\left[{ }^{3} \mathrm{H}\right]$ palmitoylHlyA was digested with endoproteinase Lys-C (12). The resulting peptides were resolubilized, first in acidic aqueous acetonitrile, which recovered $15 \%$ of the total radioactivity, and then a second time in the presence of guanidinium chloride $(\mathrm{GnCl})$, which recovered all of the remaining $85 \%$ (13). The two samples obtained were fractionated on a reversed-phase high-performance liquid chromatography (HPLC) C8 column (13) (Fig. 3). Each fractionation gave one major ${ }^{3} \mathrm{H}$ peak, the retention times of which differed substantially, which indicated that the peptides were of different size or stoichiometry of substitution or both. Recovery of ${ }^{3} \mathrm{H}$ from the first and second HPLC runs was 78 and $89 \%$, respectively, which confirmed that the relative abundance of the labeled peptides was an approximate reflection of the extent of in vitro labeling of the two sites in the intact protoxin.

Mass spectrometry (14) of HPLC peptide peak 1 (two fractions, 184 pmol of ${ }^{3} \mathrm{H}$ ) revealed that it contained a predominant species of molecular mass $1630.2 \pm 0.8$ daltons, termed peptide 1 , and that HPLC peak 2 (one fraction, $477 \mathrm{pmol}$ of ${ }^{3} \mathrm{H}$ ) con- 